\title{
30 Ubiquitous mitochondria among anaerobes
}

The discovery of hydrogenosomes (Lindmark and Müller 1973) initially had no impact on endosymbiotic theories for the origin of mitochondria, except for the occasional suggestion that hydrogenosomes might have descended from a different endosymbiont than the mitochondrion did (Whatley et al. 1979). The RNA-based revolution in microbial taxonomy spearheaded by Woese and colleagues had Giardia, Trichomonas, Entamoeba, and microsporidians branching early in eukaryote evolution, basal to mitochondrial lineages. At the time, this notion matched with cytological observations that these parasites were structurally simple eukaryotic species, intermediate forms from the prokaryote-to-eukaryote transition. That resulted in the creation of a paraphyletic group following the idea of primitive eukaryotes under a common formal name Archezoa (Cavalier-Smith 1983a). Archezoa gave the presumed primitive anaerobes in the standard model a formal name and rank. The representatives of archezoa were thought to lack mitochondria because they had branched off from the mainstream of eukaryotic evolution before the endosymbiotic event with the $\alpha$-proteobacterium occurred that gave rise to the mitochondrion. Note that the term $\alpha$-proteobacteria was not introduced until the late 1980s (Stackebrandt et al. 1988), before that they were called purple nonsulfur bacteria (John and Whatley 1975). The species of archezoa were considered as contemporary descendants of phagocyting primitively amitochondriate cells with nuclei, direct descendants of the host that acquired the $\alpha$-proteobacterial endosymbiont (Cavalier-Smith 1983b). Margulis' version of the standard model had the phagotroph arising from an archaeal-spirochaete symbiosis (Margulis et al. 2000). Doolittle's (1998) version of the standard model did not specify the mechanism by which the phagotroph arose. There was a time when people were quite confident in the standard model that had eukaryotes arising via point mutation from archaea, becoming phagocytotic for some reason. Only recently has anyone even inspected, from the standpoint of physiology, the assumption that a prokaryote could become phagocytotic via point mutation. The idea does not work because a phagocytotic prokaryote would be digesting its bioenergetic membrane in an attempt to gain energy (Martin et al. 2017), the origin of phagocytosis only works if a cell already has mitochondria.

The problems with the archezoa idea were severalfold. First, the trees were wrong. The lineages thought to be archezoa were branching in the wrong place, artefactually deep (Stiller and Hall 1999; Philippe et al. 2000a). Second, the archezoa all turned out to have organelles of mitochondrial origin after all (van der Giezen 2009). Third, aerobes and anaerobes interleaved in phylogenies (Embley and Martin 2006). Aerobic, anaerobic, and facultative anaerobic eukaryotic species, and their diverse forms of organelles of mitochondrial origin, were found to result from multiple independent specialization events to ecological niches with variable oxygen availabilities, within different evolutionary lineages (Müller et al. 2012). The defining trait of the 
prokaryote eukaryote divide no longer boiled down to the presence or absence of nuclei in cells; it had to be expanded by the presence of mitochondria.

The microsporidian fungi exemplify the phylogenetic problem. Using traditional phylogeny models, which presumed identical rate of evolution for all regions of compared sequences, they branched relatively early compared to other eukaryotes and their long branches split close to the long branches of prokaryotic outgroups (Leipe et al. 1993; Hashimoto et al. 1997). However, inclusion of larger amounts of sequence data in the comparison and the use of more modern phylogenetic methods led to completely different results, putting microsporidia into the kingdom of fungi within phylogenetic trees (Hirt et al. 1999; Keeling et al. 2000; James et al. 2006). The early branching of microsporidia in older phylogenetic trees of eukaryotes was, in hindsight, a phylogenetic artifact.

New genome sequences, the sequencing of large numbers of representatives of many eukaryotic lineages, including newly discovered ones, and the development of more complicated phylogenetic methods has led to a thorough reorganization of evolutionary relations among eukaryotes (Caron et al. 2017). Although the concept of archezoa fell apart, Margulis's phagocytotic mitochondrial acquisition idea at its heart - mitochondria as evolutionary indigestion - remained inertial to much literature on the topic, even as it was becoming evident that none of the organisms people had thought were archezoa were what the theory had predicted them to be (Doolittle 1998). Additional information was also provided by less apparent molecular characteristics, such as gene insertions and deletions at selected loci or fusions of particular genes (Burki 2014).

New views of phylogenetic relations among eukaryotes have emerged and continue to emerge (Caron et al. 2017). Most eukaryotic species are now classified into novel utilitarian taxa called supergroups (Baldauf 2003; Simpson and Roger 2004; Adl et al. 2005; Keeling et al. 2005; Parfrey et al. 2006). In newer phylogenetic trees of eukaryotes, amitochondriate lineages and anaerobes are interspersed among roughly six major branches, each representing one supergroup: Opisthokonta, Amoebozoa, Archaeplastida, Excavata, Chromalveolata, and Rhizaria, the terminology we use here. However, relationships between individual supergroups have not been completely resolved to date (Caron et al. 2017), and new lineages continue to be discovered. These new trees sometimes contain polytomies (multifurcations) at their base. This is due to different reasons. Some eukaryotic lineages are represented by insufficient numbers of species, which presents an obstacle for phylogenetics when the identification of very early branching lineages is the goal (Graybeal 1998). In addition, the problem of resolving deep branches in phylogenetic trees is generally severe (Penny et al. 2001; Ho and Jermiin 2004). But worse, even if molecular phylogeny worked perfectly, a substantial amount (perhaps all?) of the phylogenetic signal needed to solve the early branches in the diversification of eukaryotes might have been lost from sequence data (Embley and Martin 2006). Were that not bad enough, the genome of the last eukaryote common ancestor was replete with gene duplications (Tria et al. 2019), and such duplications 
are well known to confound attempts to get phylogenetic relationships sorted out. Note that some robust characters like the presence of plastids and mechanisms of protein import (Gould et al. 2015) are in direct conflict with molecular phylogenies. The only thing we can say for sure about molecular phylogenies is that they change in their most important aspects on a very regular basis as new data and new methods accrue.

As mentioned previously, the classification of ciliates is difficult, and one generally accepted important change has actually taken place in eukaryotic supergroups, in spite of numerous controversies and ambiguities, it concerns the Chromalveolata supergroup. Originally, it included four species-rich lineages of eukaryotes: alveolates, stramenopiles, haptophytes, and cryptomonads (Keeling 2004; Reyes-Prieto et al. 2007). However, as more and more phylogenetic data accumulated, support for the monophyletic origin of the four groups vanished (Burki et al. 2007; RodríguezEzpeleta et al. 2007; Burki et al. 2008). The phylogenetic position of haptophytes and cryptomonads became unclear and, conversely, the alveolates and stramenopiles were joined by the supergroup Rhizaria. This grouping created, on the rubble of the Chromalveolata supergroup, a new monophyletic SAR supergroup named after the included clades (the acronym contains the first letters of "stramenopiles," "alveolates," and the name of the original eukaryotic supergroup Rhizaria) (Burki et al. 2007; Burki 2014). However, the mechanism and protein machinery that the photosynthetic members of the chromalveolates (or SAR) use to import proteins into their red secondary plastids are the same (Gould et al. 2015), an observation that argues in favor of chromalveolates being correct after all and that is not subject to the vagaries of phylogenetic tree parameters and uncertainty. As a side note, we point out that the loss of support for the chromalveolates with larger amounts of data could be due to additive effects of undetected paralogy (gene duplications). The plastids of the chromalveolates are an example where endosymbiosis as an organelle-generating process produced higher level cytological structures (plastids) that have a very clear phylogenetic interpretation (single origin, recurrent reduction, occasional loss), while individual gene trees generate massively conflicting results.

Coming back to the mitochondrial family of organelles and anaerobic energy metabolism in eukaryotes, the current phylogenetic tree with five (or six) supergroups provides a completely different view than the one described in the 1980s and 1990s by the concept of archezoa. Hydrogenosomes, mitosomes, and various transition forms of the organelles of mitochondrial origin are found on different branches located throughout the phylogenetic tree representing the full breadth of eukaryotic diversity (Figure 32). Eukaryotic anaerobes are not rare, unusual, or phylogenetically primitive species occupying obscure early branches of the tree. On the contrary, their numerous representatives overlap, evolutionarily, with aerobic relatives, which indicates that aerobic and anaerobic species of eukaryotes evolved side by side, at least since ocean oxygenation at the end of Proterozoic (Mentel and Martin 2008; Lyons et al. 2014). 


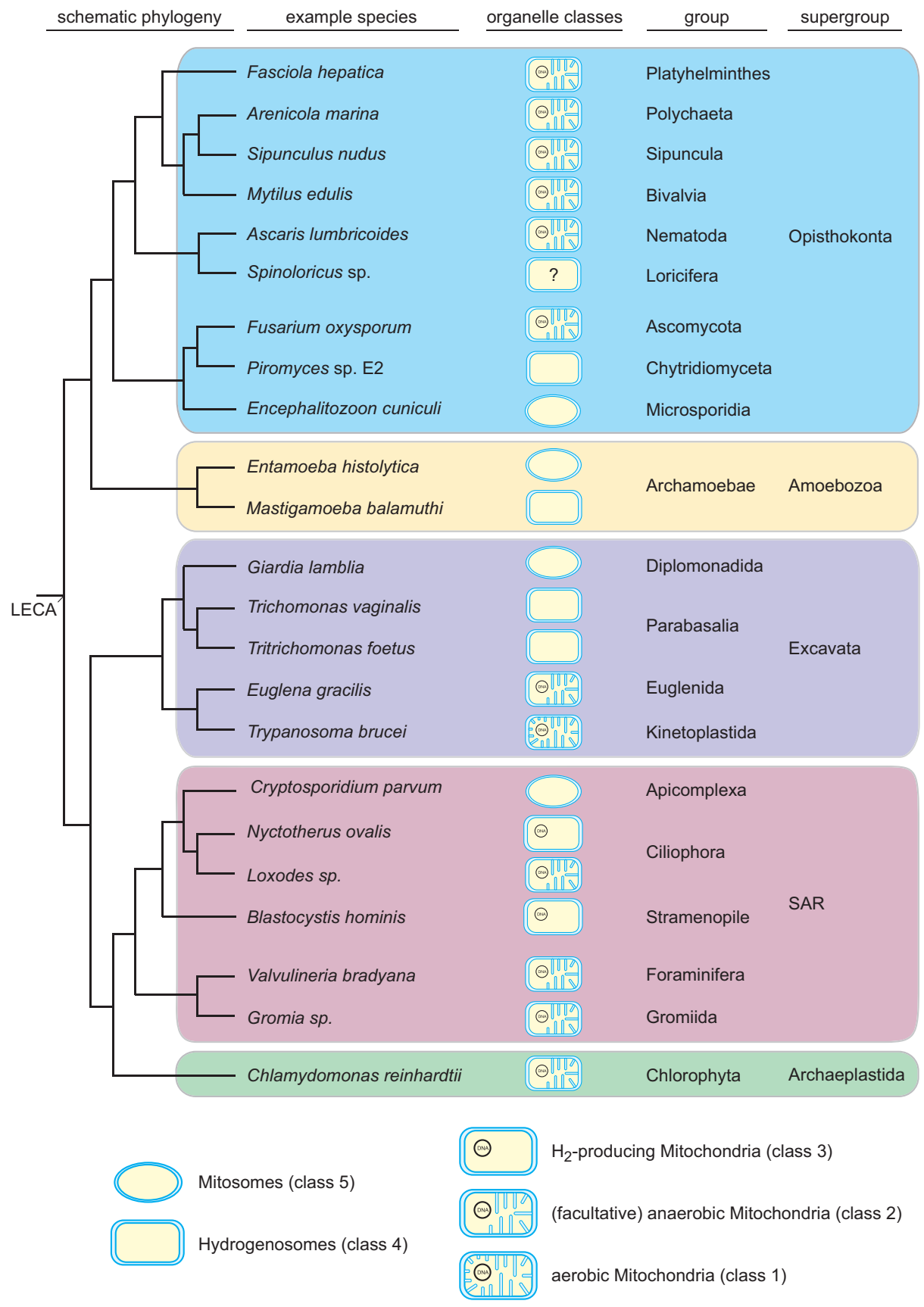

Figure 32: Anaerobic mitochondria across eukaryote supergroups. The topology of the tree reflects the five currently accepted eukaryotic supergroups (Burki 2014); the branching adheres to recent suggestions (Burki et al. 2016). The species shown are those model organisms whose anaerobic 
The changes in views about taxonomic relations among eukaryotes will continue. There has been a proposal, for instance, that the drop-outs from the Chromalveolata supergroup, haptophytes and cryptomonads, together with several minor lineages, form a new supergroup Hacrobia or clade CCTH (abbreviation contains the first letters of "cryptophyta," "centrohelida," "telonemia," and "haptophytes") (Okamoto et al. 2009). However, same as with the Chromalveolata supergroup, the monophyletic origin of all members of the club has never been confirmed; therefore, it is simpler to refer to individual lineages with unclear phylogenetic affinity. The same applies to representatives of the lineages "Picozoa," "Microhelida," or photosynthesizing rappemonads (lat. "Rappemonada") (Speijer et al. 2015). The only constant in molecular phylogenetics is that the trees always change; the organisms stay as they are, regardless of where they branch in phylogenetic trees.

An ever-growing group of small but evolutionarily relevant eukaryotic lineages has been uncovered, consisting of amoebas, amoeboflagellates, and flagellates, which have been assigned the rank of microkingdoms and cannot be included in any of the accepted supergroups (Pawlowski 2013). They are, for instance, microkingdoms of apusomonadids (lat. "Apusomonadida") and breviatids (lat. "Breviatea"), which were proposed to fuse with the Opisthokonta supergroup and form an even larger group under a new name Obazoa (Brown et al. 2013). The legitimacy of such grouping depends, though, on the root position of the eukaryotic tree, which is another phylogenetic issue that has not yet been resolved (Burki 2014). Historically, during the division of eukaryotes into six supergroups, the root of the phylogenetic tree including all eukaryotes was placed between two large groups: (1) Unikonta, which included supergroups Opisthokonta and Amoebozoa and (2) Bikonta with supergroups Archaeplastida, Excavata, Chromalveolata, and Rhizaria (later Archaeplastida, Excavata, and SAR) (Cavalier-Smith 2002). The group Unikonta was later renamed to Amorphea (Adl et al. 2012) and the position of the tree root between Unikonta and Bikonta (Embley and Martin 2006) indicated in Figure 32 is still the most popular (Burki 2014).

Multiple hypotheses have appeared, though, with an alternative positioning of the root, one that splits eukaryotic life into two groups named Opimoda and Diphoda (Derelle et al. 2015). However, that division of eukaryotes would dissolve the Excavata as a supergroup because one of its three main groups, Discoba, would

Figure 32 (continued)

energy metabolism has been described in detail, in most cases. Their distribution in the phylogenetic tree indicates that anaerobic eukaryotic species are not isolated from aerobic eukaryotes, as they are found in all supergroups and cannot be considered to be evolutionarily primitive or descendants of primitive eukaryotes. As the only obligate aerobic species, the protist Trypanosoma brucei is shown. Presence of a genome in individual organelles is indicated by a circle inscribed "DNA." Sizes of the different organelles of mitochondrial origin are not shown to scale. Identity of mitochondria of the animal phylum Loricifera has not been clarified so far. Abbreviation: LECA, last eukaryotic common ancestor. 
be classified to the group Diphoda, while another main group Malawimonadida would belong to the group Opimoda. The phylogenetic position of the third main group, Metamonada, has not been resolved convincingly (Speijer et al. 2015). These groupings change the classification of the organisms, but not their physiology. The last eukaryote common ancestor, LECA, had hundreds of gene duplications in its genome (Tria et al. 2019). Duplications generate differential gene loss in different lineages, which generates paralogy, which in turn makes the identification of the root in the eukaryote tree with gene phylogenies difficult at best. The phylogenetic distribution of the duplications themselves places the eukaryotic root on the branch bearing the Excavata (Tria et al. 2019). In any case, topological adjustments of the eukaryotic phylogenetic tree do not change the central finding that anaerobic eukaryotes are found throughout the breadth of eukaryotic diversity on the Earth, mutually interlacing with their aerobic relative species, from which they are not distinguished in a phylogenetic manner, regardless of where the root lies. 